\title{
Hemangioma intraóseo primario de la órbita: a propósito de dos casos
}

\author{
E. Torres-Carranza; A. García-Perla; P. Infante-Cossío; M. Acosta-Feria; R. Belmonte-Caro y J.L. Gutiérrez-Pérez
}

Servicio de Cirugía Oral y Maxilofacial. Hospital Universitario Virgen del Rocío. Sevilla.

\section{Resumen}

El hemangioma intraóseo primario, aunque rara vez afecta a los huesos de la órbita, se debe tener en cuenta en el diagnóstico diferencial en aquellos pacientes que presenten una masa fija en la órbita o signos de proptosis progresiva no dolorosa. Para el planteamiento terapéutico es crítico valorar la localización exacta y la extensión de la lesión mediante TC y RNM, y establecer si tiene un origen vascular, pues un manejo inadecuado puede dar lugar a una hemorragia severa. El tratamiento quirúrgico se indica ante una proptosis progresiva o una deformidad estética. Se presentan dos casos de pacientes con hemangiomas primarios intraóseos del reborde supralateral de la órbita. Se realizó una extirpación con un margen óseo sano y se reconstruyó la órbita de forma inmediata mediante injertos de calota fijados con placas reabsorbibles. En el seguimiento a 3 años no ha habido evidencia de recurrencia local, y los resultados funcionales y estéticos obtenidos han sido muy satisfactorios.

PALABRAS CLAVE: Órbita. Hemangiomas. Intraóseo. Tumor. Incisión bicoronal. Injerto óseo. Placa reabsorbible.

Primary intraosseous hemangioma of the orbit: Report of two cases

\section{Summary}

Primary intraosseous hemangioma, though rarely affect the bones of the orbit, should be considered in the differential diagnosis when a patient presents an enlarging mass fixed to the bone in the orbit or signs of progressive painless proptosis. Assessing the exact site and extent of the hemangioma by means of a CT scan and MRI and establishing the vascular origin of the lesion, is critical in the therapeutical planning, since an

Recibido: 10-10-06. Aceptado: 17-11-06 inadequate management may result in a severe hemorrhage. Progressive proptosis and contour deformity require surgical treatment. Two cases of patients with primary intraosseus hemangioma of the supra-lateral orbital rim are reported. A bony healthy margin excision was performed, followed by immediate reconstruction of the orbit with calvarial bone grafts fixed with resorbable plates. After three-year follow-up there have not been evidence of local recurrence, and the functional and aesthetic results obtained have been very satisfactory.

KEY WORDS: Orbit. Hemangiomas. Intraosseous. Tumour. Bicoronal incision. Bone graft. Resorbable plate.

\section{Introducción}

Los hemangiomas y las malformaciones vasculares son las neoplasias más frecuentes en los tejidos blandos de la órbita ${ }^{16}$. Sin embargo, los hemangiomas de localización primaria en hueso representan menos del $1 \%$ de todas las neoplasias óseas. Más del 50\% aparecen en las vértebras y en el cráneo, afectando en esta última localización sobre todo al frontal y parietal. En los huesos de la órbita se diagnostican alrededor del 5\% ${ }^{1}$. Los hemangiomas primarios intraóseos orbitarios son neoplasias benignas que se presentan como una masa dura que en su lento crecimiento pueden causar una deformidad facial significativa, así como dolores de cabeza y problemas oculares (proptosis, diplopia y pérdida visual $)^{5,12,19}$. Los localizados en la región superolateral pueden crecer hacia la fosa craneal media posteriormente o afectar al techo de la órbita superiormente, y por tanto pueden llegar a dar síntomas con más frecuencia ${ }^{9}, 13$. $\mathrm{Su}$ diagnóstico etiológico generalmente suele ser difícil pues rara vez muestran signos clínicos característicos que sugieran una lesión vascular. El manejo terapéutico debe consistir en la escisión local de la lesión con un margen de hueso sano, y la reconstrucción inmediata de la órbita con injertos de hueso autógeno para minimizar la deformidad estética y funcional.

El objetivo de este trabajo ha sido presentar nuestra 
experiencia clínica y terapéutica en dos pacientes afectos de hemangiomas primarios de la órbita, con especial consideración en los aspectos reconstructivos y los resultados estéticos y funcionales obtenidos.

\section{Casos clínicos}

Caso 1. Un varón de 54 años de edad fue remitido para estudio de una tumoración indolora en región supraorbitaria derecha aparecida un año antes, la cual había evolucionado con un aumento lento y progresivo, provocando proptosis en el globo ocular derecho. La historia clínica del paciente no refería antecedentes de interés, ni traumatismos previos. No presentaba alteraciones oculares, neurológicas ni sistémicas. El paciente aportaba una TC inicial (Fig. 1) que mostraba una zona de expansión ósea con marcada reacción perióstica en el reborde lateral de la órbita derecha, que había sido informada como condrosarcoma o lesión metastásica de tumor primario de origen desconocido. La TC toraco-abdominal no evidenciaba lesión alguna. En la exploración clínica se comprobó una masa de consistencia dura, no desplazable y discretamente dolorosa a la presión en el reborde supero-lateral orbitario derecho, de tamaño aproximado de 4 × $3 \mathrm{~cm}$. La piel suprayacente era normal. Se solicitó una RNM que reveló una lesión ósea en la pared superolateral del la órbita derecha con áreas hiper e hipodensas.

Se accedió a la lesión mediante un abordaje con incisión bicoronal, y se tomó una biopsia intraoperatoria que fue informada como compatible con un hemangioma intraóseo. La masa fue extirpada en bloque con un margen de seguridad de hueso sano, incluyendo parte del reborde superior y pared lateral de la órbita. El defecto óseo fue reparado con un injerto óseo monocortical del hueso parietal, y fue fijado con una placa y tornillos reabsorbibles (Fig. 2). El sangrado

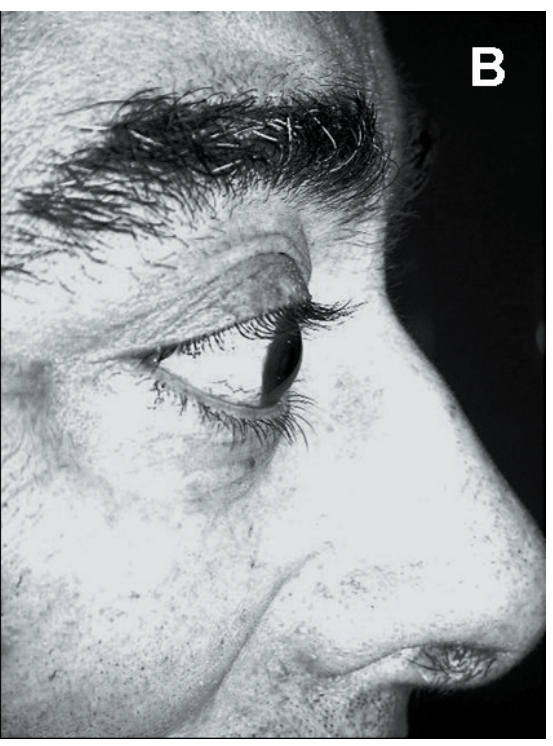

Figura 1. Caso 1. (A) TC axial inicial que muestra una zona de expansión ósea en el reborde lateral de la órbita derecha con protusión del ojo $y$ (B) aspecto clínico de proptosis del globo ocular.

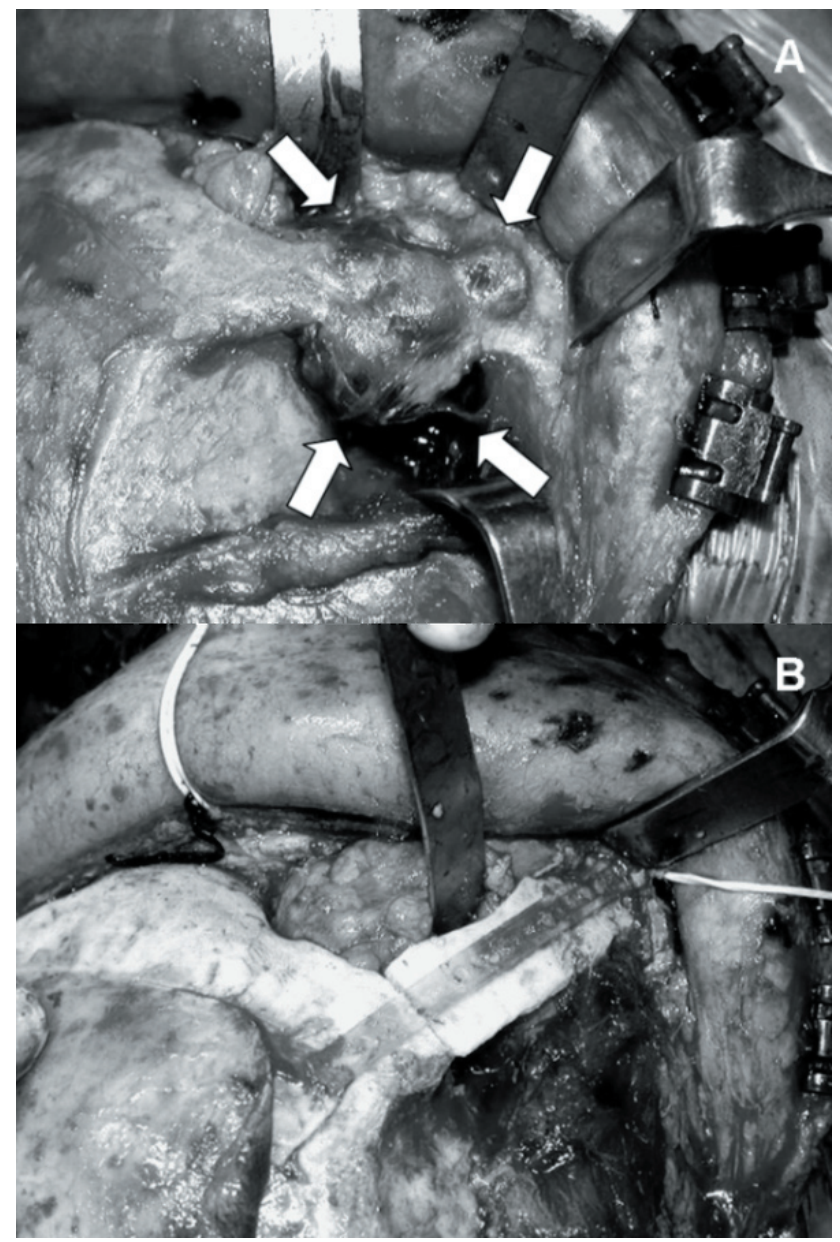

Figura 2. Caso 1. (A) Abordaje quirúrgico al hemangioma intraóseo (flechas) y (B) reparación del defecto tras la extirpación en bloque con un injerto óseo de calota fijado con placa y tornillos reabsorbibles. 


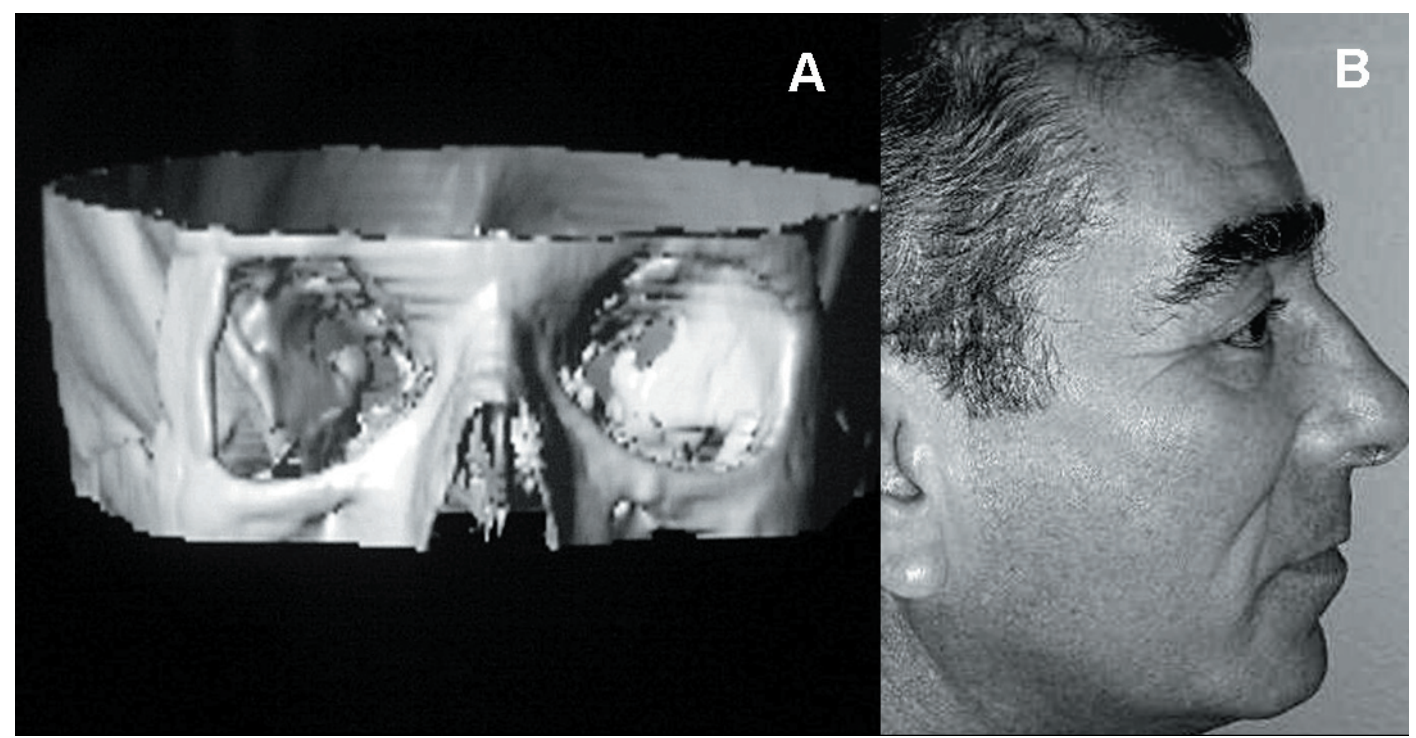

Figura 3. Caso 1. Seguimiento a los 4 años: (A) TC-3D que evidencia la incorporación del injerto óseo y (B) resultado estético.

intraoperatorio fue mínimo. La evolución postoperatoria cursó sin incidencias. El paciente fue dado de alta del hospital en 5 días. El informe histológico definitivo confirmó el diagnóstico de hemangioma cavernoso intraóseo. Tras un seguimiento del paciente durante 4 años, no se han detectado signos de recurrencia local de la lesión. La incorporación del injerto óseo ha sido adecuada, y los resultados estéticos y funcionales, satisfactorios (Fig. 3).

Caso 2. Un varón de 37 años de edad, sin antecedentes personales de interés ni traumatismos previos, acudió por presentar una tumoración en región superolateral orbitaria izquierda de un año de evolución, que progresivamente le había causado una desviación hacia abajo del globo ocular, y en el último mes, visión doble. A la exploración se apreció una masa dura, inmóvil y no dolorosa, con un tamaño aproximado de 2,5 x $1 \mathrm{~cm}$, localizada en el reborde orbitario superolateral izquierdo, al que estaba adherido. La exploración oftalmológica y neurológica fue normal. La RNM evidenció una lesión bien definida en el reborde orbitario izquierdo que afectaba al espacio extraconal (Fig. 4). La secuencia en T2

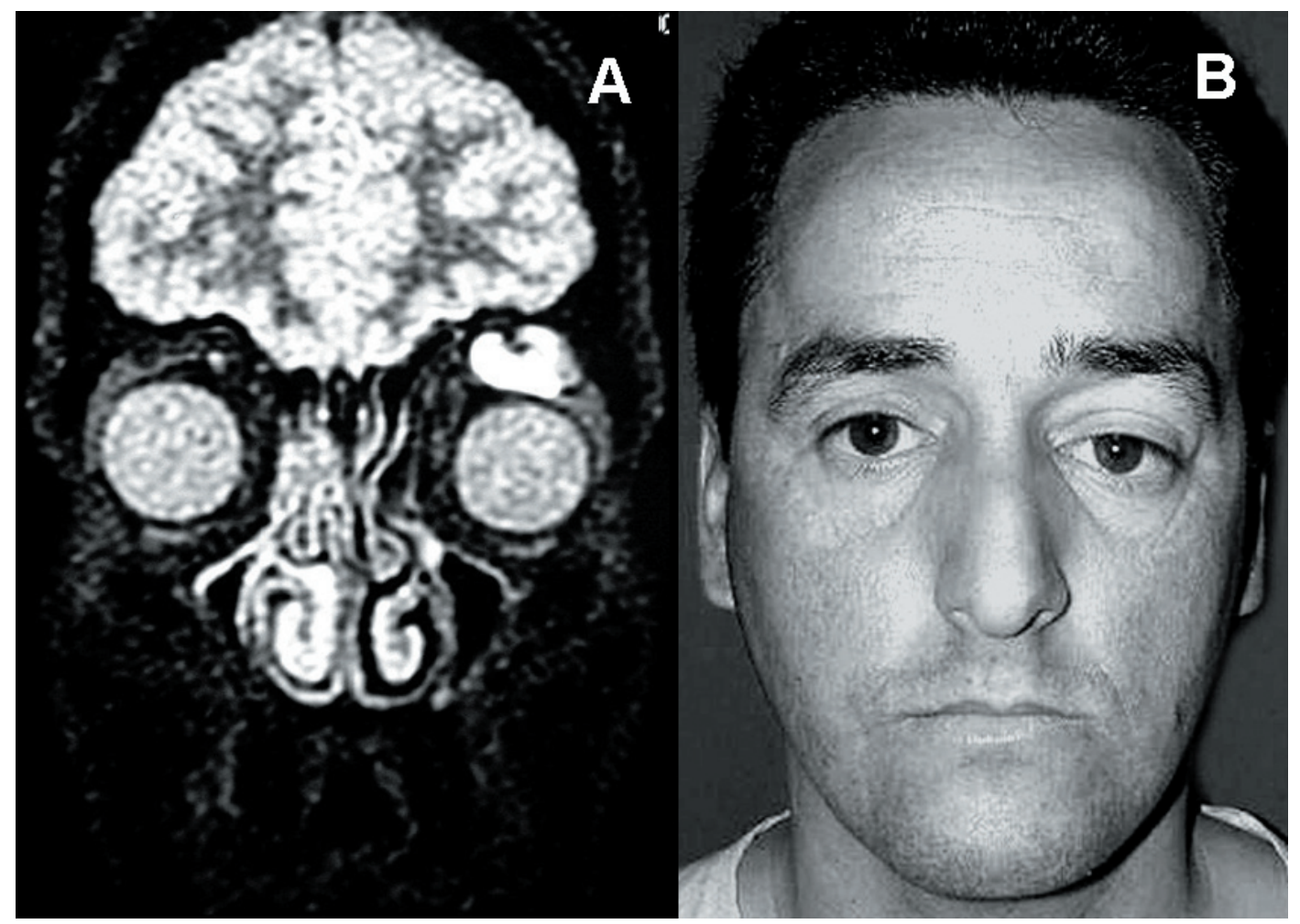

Figura 4. Caso 2. (A) RNM coronal donde se observa una lesión en el reborde orbitario izquierdo que afecta al espacio extraconal y (B) distopia inicial evidente. 

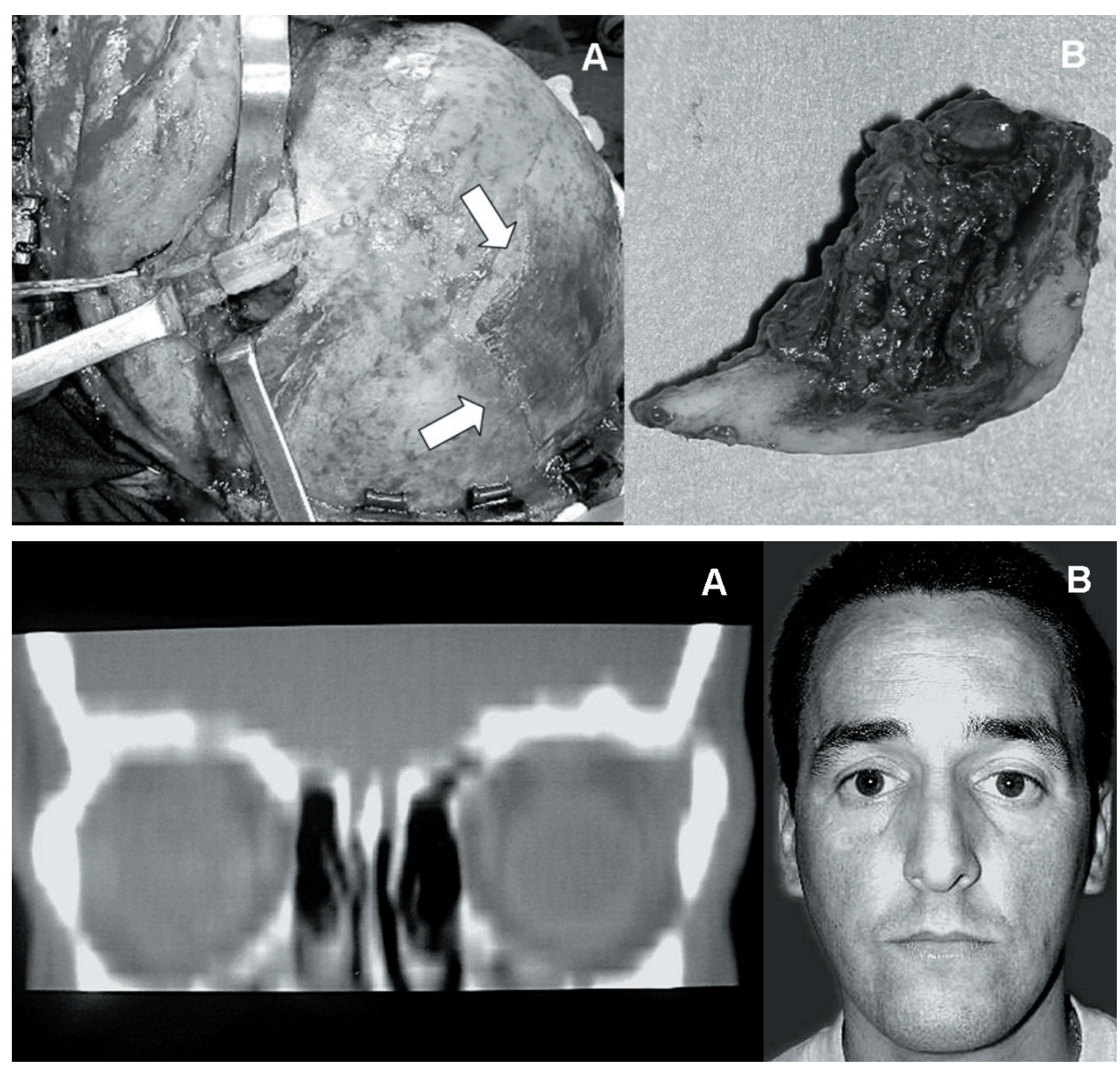

Figura 5. Caso 2. (A)

Detalle de la reparación de la continuidad ósea con un injerto óseo monocortical y placa reabsorbible, y de la zona donante parietal (flechas) y (B) pieza quirúrgica del hemangioma intraóseo.

mostraba una señal de intensidad no homogénea característica de los productos de degradación sanguíneos, sugestivo de hemangioma intraóseo. La TC mostraba la existencia de una masa sólida y ovalada, de 22 × 21 x $10 \mathrm{~mm}$ en el espacio superolateral extraconal, que producía una moderada proptosis y descenso del globo ocular izquierdo. La masa presentaba una calcificación central que captaba contraste, compatible con un hemangioma cavernoso intraóseo.

A través de una incisión bicoronal se abordó el reborde orbitario superolateral y se procedió a extirpar la masa en bloque con una porción de hueso sano adyacente. El defecto fue reparado de forma inmediata con un injerto óseo monocortical tomado del hueso parietal, que se fijó con una placa y tornillos reabsorbibles (Fig. 5). La evolución postoperatoria cursó sin incidencias. El paciente fue dado de alta hospitalaria a las 72 horas. El diagnóstico anatomopatológico correspondió con un hemangioma cavernoso intraóseo. El resultado estético y funcional tras un seguimiento de 3 años ha sido satisfactorio. No hay signos de recurrencia local de la lesión, y la incorporación del injerto óseo ha sido adecuada (Fig. 6).

\section{Discusión}

Los hemangiomas óseos se clasifican en tres tipos: cavernoso, capilar y mixto ${ }^{4}$. La mayor parte de los diagnosticados en cabeza y cuello son del tipo cavernoso, como así sucedió en los dos casos presentados en esta serie. La patogenia de esta neoplasia es desconocida. Algunos las consideran como malformaciones vasculares del desarrollo, aunque también se ha implicado traumatismos pre$\operatorname{vios}^{6,16}$, antecedentes que no fueron recogidos en ninguno de los dos casos presentados. Los hemangiomas intraóseos son tres veces más frecuentes en mujeres que en hombres. Son propios de la $4^{\circ}-5^{\circ}$ década, aunque pueden aparecer en cualquier edad ${ }^{19}$.

El diagnóstico clínico y radiológico de un hemangioma intraóseo plantea en ocasiones serias dificultades, como se comprueba con el caso 1. Establecer el origen vascular de la lesión puede ser crítico, ya que una biopsia ósea o 
curetaje inadecuado puede dar lugar a una severa hemorra$\mathrm{gia}^{13}$. La radiología simple muestra una imagen diagnóstica característica, generalmente de rarefacción ósea con márgenes bien definidos ${ }^{1}$. A veces pueden presentar patrones diagnósticos concretos (estriaciones finas, reticulaciones tipo panal de abejas, imágenes de rayos de sol en la cortical ósea, etc.), que no se comprobaron en los casos presentados. La TC es útil para conocer la localización y extensión de la neoplasia. La RNM muestra una señal incrementada en las imágenes en T1 y T2. El tejido blando de la lesión causa incremento de la señal en $\mathrm{T} 1^{15}$, y estancamiento sanguíneo y flujo lento en $\mathrm{T} 2^{1}$. Sin embargo, a pesar de sus manifestaciones radiológicas específicas, la mayoría de estas neoplasias sólo pueden ser diagnosticadas definitivamente tras su extirpación quirúrgica ${ }^{8,11}$. La angiografía preoperatoria no es necesaria generalmente, pues estas lesiones no suelen tener un aporte sanguíneo reconocible ${ }^{4}$, y no fue utilizada en estos pacientes.

El diagnóstico diferencial de las lesiones óseas primarias de lento crecimiento en la órbita es muy amplio, ya que existen gran número de lesiones de presentación clínica y radiográfica similar a los hemangiomas óseos. Entre éstas se incluyen las displasias fibrosas, quistes óseos aneurismáticos, tumor de células gigantes, osteomas, quistes dermoides, meningiomas, metástasis y tumores malignos de la base del cráneo como el condrosarcoma, mieloma múltiple o sarcomas osteogénicos. En el caso 1, la sospecha inicial fue de un tumor maligno o metastásico, mientras el diagnóstico del caso 2 fue compatible radiológicamente con el hemangioma.

La cirugía debe consistir en una escisión local del tumor en bloque con un margen de hueso sano cuando el diagnóstico de hemangioma intraóseo se conoce pre o intraoperatoriamente ${ }^{7,10}$, tal y como se hizo en los dos casos presentados. De esta forma, la resección de un margen de hueso sano permite extirpar completamente la lesión y reducir el sangrado operatorio. El curetaje de la lesión conlleva el riesgo de hemorragia incontrolable ${ }^{14}$. Cuando no hay compromiso ocular (proptosis) ni estético, algunos autores recomiendan la observación como medida alternativa $^{3}$. La resección parcial con una osteotomía superficial conduce a la recurrencia en un alto porcentaje de casos, aunque otros autores la indican como una técnica simple sólo para corregir la deformidad ósea ${ }^{4}$. Si el diagnóstico es incierto o se sospecha malignidad, la resección ósea debe ser mayor con objeto de obtener márgenes libres de tumor ${ }^{16}$. Si la lesión se localiza en la supraórbita, puede hacerse necesaria una craniotomía con posterior reconstrucción del defecto óseo. La embolización supraselectiva en las 24-72 horas previas a la cirugía y la ligadura de la arteria carótida externa reduce significativamente el sangrado intraoperatorio, y puede ser efectivo en aquellas lesiones de gran tamaño y rica vascularización ${ }^{6}$. El tratamiento con radioterapia de estos tumores puede ser empleado en casos irresecables, pues se ha demostrado su utilidad para detener su crecimiento ${ }^{17}$.

En los dos casos presentados en este trabajo, las lesiones pudieron ser resecadas con un margen óseo sano y la reconstrucción de la órbita se llevó a cabo de forma inmediata. La reconstrucción del defecto óseo orbitario con injertos autógenos de calota puede ser ideal, pues elimina los riesgos a largo plazo de los materiales de reconstrucción sintéticos, y se ha demostrado que posee una óptima incorporación y baja reabsorción con el tiempo, con mínimos efectos secundarios ${ }^{18}$. La fijación del injerto autógeno de hueso con placas de osteosíntesis reabsorbibles muestra la ventaja de constituir un material biocompatible y adaptable, que ofrece una estabilidad adecuada para permitir la cicatrización ósea pero que posteriormente se reabsorbe, evitando los problemas que pueden presentar las placas de titanio con el tiempo ${ }^{2}$. La evolución y el seguimiento clínico de los pacientes de esta serie ha sido muy satisfactoria. No ha habido evidencia de recurrencia local del hemangioma, y los resultados obtenidos desde el punto de vista funcional y estético demuestran que el tratamiento de elección de esta rara neoplasia debe incluir la resección en bloque y la reconstrucción inmediata de la órbita con injerto autógeno de hueso.

\section{Bibliografía}

1. Banerji, D., Inao, S., Sugita, K., Kaur, A., Chhabra, D.K.: Primary intraosseous orbital hemangioma: A case report and review of the literature. Neurosurg 1994; 35: 1131-1134.

2. Bell, R.B., Kindsfater, C.S.: The use of biodegradable plates and screws to stabilize facial fractures. J Oral Maxillofac Surg 2006; 64: 31-39.

3. Charles, N.C., Lisman, R.D.: Intraosseous hemangioma of the orbit. Ophthalmic Surg Lasers 2002; 33: 326-328.

4. Cheng, N.C., Lai, D.M., Hsie, M.H., Liao, S.L., Chen, Y.B.: Intraosseous hemangiomas of the facial bone. Plast Reconstr Surg 2006; 117: 2366-2372.

5. Colombo, F., Cursiefen, C., Hofmann-Rummelt, C., Holbach, L.M.: Primary intraosseous cavernous hemangioma of the orbit. Am J Ophthalmol 2001; 131: 151-152.

6. Cuesta Gil, M., Navarro-Vila C.: Intraosseous hemangioma of the zygomatic bone. A case report. Int J Oral Maxillofac Surg 1992; 21: 287-291.

7. Hwang, K.: Intraosseous hemangioma of the orbit. J Craniofac Surg 2000; 11: 386-387.

8. Kolasa, P., Januszalwasiak, Kordek, R, Boronski, K.: Intraosseous orbital hemangioma. Folia Neuropathol 2001; 39: 37-39.

9. Leibovitch, I., Dray, J.P., Leibovitch, L., Brazowski, E.: Primary intraosseous hemangioma of the zygomatic bone. Plast Reconstr Surg 2003; 111: 519-521. 


\section{Hemangioma intraóseo primario de la órbita: a propósito de dos casos}

10. Moore, S.L., Chun, J.K., Mitre, S.A., Som, P.M.: Intraosseous hemangioma of the zygoma: CT and MR findings. Am J Neuroradiol 2001; 22: 1383-1385.

11. Okada, A.A., Shore, J.W., Rubin, P.A.D.: Periorbital intraosseous hemangiomas. Int Ophthalmol Clin 1992; 32: 111-122.

12. Relf, S.J., Bartley, G.B., Unni, K.K.: Primary orbital intraosseous hemangioma. Ophthalmology 1991; 98: 541547.

13. Rios Dias, G.D., Velasco Cruz, A.A.: Intraosseous hemangioma of the lateral orbital wall. Ophthal Plast Reconstr Surg 2004; 20: 27-30.

14. Savastano, G., Russo, A., Dell'Aquila, A.: Osseous hemangioma of the zygoma: A case report. J Oral Maxillofac Surg 1997; 55: 1352-1356.

15. Sweet, C., Silbergleit, R., Mehta, B.: Primary intraosseous hemangioma of the orbit: CT and MR appearance. Am J Neuroradiol 1997; 18: 379-381.

16. Tang Chen, Y.B., Wornom, I.L., Whitaker, L.A.: Intraosseous vascular malformations of the orbit. Plast
Neurocirugía 2007; 18: $320-325$

Reconstr Surg 1991; 87: 946-949.

17. Taylan, G., Yildirim, S., Gideroglu, K., Akoz, T.: Conservative approach in a rare case of intrazygomatic hemangioma. Plast Reconstr Surg 2003; 112: 1490-1492.

18. Zins, J.E., Turegun, M.C., Hosn, W., Bauer, T.W.: Reconstruction of intraosseous hemangiomas of the midface using split calvarial bone grafts. Plast Reconstr Surg 2006; 117: 948-953.

19. Zucker, J.J., Levine, M.R., Chu, A.: Primary intraosseous hemangioma of the orbit: Report of acase and review of the literature. Ophthal Plast Reconstr Surg 1989; 5: 247-255.

Torres-Carranza, E.; García-Perla, A.; Infante-Cossío, P.; Acosta-Feria, M.; Belmonte-Caro, R.; Gutiérrez-Pérez, J.L.: Hemangioma intraóseo primario de la órbita: a propósito de dos casos. Neurocirugía 2007; 18: 320-325.

Correspondencia postal: Dr. Pedro Infante Cossío. Servicio de Cirugía Maxilofacial. Hospital Universitario Virgen del Rocío. Av. Manuel Siurot s/n. 41013-Sevilla (Spain) 